PROCEEDINGS OF THE

AMERICAN MATHEMATICAL SOCIETY

Volume 133, Number 2, Pages 587-594

S 0002-9939(04)07702-0

Article electronically published on August 25, 2004

\title{
ON THE FIRST HODGE EIGENVALUE OF ISOMETRIC IMMERSIONS
}

\author{
ALESSANDRO SAVO
}

(Communicated by Jozef Dodziuk)

\begin{abstract}
We give an extrinsic upper bound for the first positive eigenvalue of the Hodge Laplacian acting on $p$-forms on a compact manifold without boundary isometrically immersed in $\mathbf{R}^{n}$ or $\mathbf{S}^{n}$. The upper bound generalizes an estimate of Reilly for functions; it depends on the mean value of the squared norm of the mean curvature vector of the immersion and on the mean value of the scalar curvature. In particular, for minimal immersions into a sphere the upper bound depends only on the degree, the dimension and the mean value of the scalar curvature.
\end{abstract}

\section{The Main estimate}

Let $M^{n}$ be a compact, oriented Riemannian manifold without boundary and $f: M^{n} \rightarrow \mathbf{R}^{n+m}$ an isometric immersion. Let $\mu_{1}(M)$ denote the first positive eigenvalue of the Laplacian on $M$. Reilly proved in [ $\mathbb{R}$ ] that

$$
\mu_{1}(M) \leq \frac{n}{\operatorname{Vol}(M)} \int_{M}\|H\|^{2}
$$

where $H$ is the mean curvature vector of the immersion.

The scope of this note is to extend (1.1) to the Laplacian $\Delta_{p}$ acting on differential $p$-forms on the manifold $M$. For the main facts about $\Delta_{p}$ (also denoted simply by $\Delta$ and often called the Hodge Laplacian of $M$ ) we refer to [C].

By definition,

$$
\Delta=d \delta+\delta d
$$

where $d$ is the exterior derivative and $\delta=(-1)^{n(p+1)+1} \star d \star$ is the codifferential, $\star$ being the Hodge operator. $\Delta_{p}$ has a discrete, nonnegative spectrum, and its kernel is known to be isomorphic to the real $p$-th de Rham cohomology space of $M$; we denote by $\mu_{1, p}(M)$ its first positive eigenvalue.

Let $\mu_{1, p}^{\prime}$ (resp. $\mu_{1, p}^{\prime \prime}$ ) denote the first (positive) eigenvalue of $\Delta_{p}$ when restricted to the space of exact (resp. co-exact) forms (these spaces are preserved by $\Delta_{p}$ because it commutes with both $d$ and $\delta$ ). The Hodge decomposition theorem implies that

$$
\mu_{1, p}=\min \left\{\mu_{1, p}^{\prime}, \mu_{1, p}^{\prime \prime}\right\},
$$

Received by the editors January 22, 2003.

2000 Mathematics Subject Classification. Primary 58J50; Secondary 53C42.

Key words and phrases. Laplacian on $p$-forms, first eigenvalue, isometric immersions, minimal immersions.

(C)2004 American Mathematical Society Reverts to public domain 28 years from publication 
and, by differentiating eigenforms, $\mu_{1, p}^{\prime \prime}=\mu_{1, p+1}^{\prime}$. Hence it is enough to give an upper bound of $\mu_{1, p}^{\prime}$ for all $p$. Then we have:

Theorem 1.1. Let $f: M^{n} \rightarrow \mathbf{R}^{n+m}$ be an isometric immersion. For all $p=$ $1, \ldots, n$ :

$$
\mu_{1, p}^{\prime}(M) \leq \frac{1}{\operatorname{Vol}(M)} \int_{M}\left[p n\|H\|^{2}-\frac{p(p-1)}{n(n-1)} \text { scal },\right.
$$

where scal is the scalar curvature of $M^{n}$. Equality holds for the canonical immersion $\mathbf{S}^{n} \rightarrow \mathbf{R}^{n+1}$.

By the Hodge duality (induced by the $\star$ operator) one has

$$
\mu_{1, p}^{\prime}=\mu_{1, n-p}^{\prime \prime}=\mu_{1, n-p+1}^{\prime} .
$$

Hence Theorem 1.1 gives two (generally) different upper bounds for the same eigenvalue. However, using the Gauss lemma (see (2.8)) it can be checked that, if $p \leq \frac{n+1}{2}$, our upper bound in degree $p$ is better than the one in degree $n-p+1$, and so it is convenient to assume (without loss of generality) that $p \leq \frac{n+1}{2}$.

For example, since

$$
\mu_{1}(M)=\mu_{1,1}^{\prime}(M)=\mu_{1, n}^{\prime}(M)
$$

Theorem 1.1 gives the Reilly inequality 1.1 for $p=1$, while for $p=n$ (see also (2.8) ) it gives the following estimate, weaker than Reilly's and previously obtained in $[\mathrm{B}-\mathrm{W}]$ :

$$
\mu_{1}(M) \leq \frac{1}{\operatorname{Vol}(M)} \int_{M}\|S\|^{2}
$$

where $\|S\|^{2}$ is the squared length of the second fundamental form of the immersion (which is always greater than or equal to $n\|H\|^{2}$ ).

By composing a given immersion of $M$ in $\mathbf{S}^{n+m}$ with the canonical immersion of $\mathbf{S}^{n+m}$ in $\mathbf{R}^{n+m+1}$, one gets:

Corollary 1.2. Let $M^{n} \rightarrow \mathbf{S}^{n+m}$ be an isometric immersion. Then:

$$
\mu_{1, p}^{\prime}(M) \leq p n+\frac{1}{\operatorname{Vol}(M)} \int_{M}\left[p n\|H\|^{2}-\frac{p(p-1)}{n(n-1)} \text { scal }\right] .
$$

In particular, if the immersion is minimal, then

$$
\mu_{1, p}^{\prime}(M) \leq p n-\frac{p(p-1)}{n(n-1)} \cdot \frac{1}{\operatorname{Vol}(M)} \int_{M} \text { scal }
$$

Remark 1.3. The immersed manifold $M$ in this paper is assumed without boundary. We refer to [G-S] for a lower bound of the gap $\mu_{1, p}^{\prime \prime}-\mu_{1, p}^{\prime}$, leading to other kinds of estimates of the first Hodge eigenvalue which are valid also when the manifold has nonempty boundary.

\section{SCHEME OF PROOF AND BASIC LEMMAS}

The Hodge Laplacian is associated to the quadratic form

$$
Q(\omega, \omega)=\int_{M}\|d \omega\|^{2}+\|\delta \omega\|^{2} .
$$

Restricting to the space of exact forms, one has Rayleigh's min-max principle:

$$
\mu_{1, p}^{\prime}(M)=\inf \left\{\frac{\int_{M}\|\delta \omega\|^{2}}{\int_{M}\|\omega\|^{2}}, \omega \in d \Lambda^{p-1}(M) \backslash\{0\}\right\} .
$$


Let us describe the exact test $p$-forms that will lead to the result. Consider the family of all parallel unit vector fields on $\mathbf{R}^{n+m}$. This family can be identified with $\mathbf{S}^{d}$, where $d=n+m-1$. Any such field $\bar{V}$ can be split, at any point $x \in M$ :

$$
\bar{V}=V+V^{\perp},
$$

where $V$ is the orthogonal projection of $\bar{V}$ on $T_{x} M$ and $V^{\perp} \in T_{x} M^{\perp}$. We denote by $\beta_{V}$ the dual 1-form of $V$ in $M$. It is exact; in fact,

$$
\beta_{V}=d\left(\rho_{\bar{V}} \circ f\right)
$$

where $\rho_{\bar{V}}: \mathbf{R}^{n+m} \rightarrow \mathbf{R}$ is the distance, taken with sign, from the hyperplane through the origin orthogonal to $\bar{V}$.

For any $\bar{V}_{1}, \ldots, \bar{V}_{p} \in \mathbf{S}^{d}$ we consider the (exact) test-form

$$
\beta_{V_{1}} \wedge \cdots \wedge \beta_{V_{p}}
$$

and so, by the min-max principle (1.1):

$$
\mu_{1, p}^{\prime}(M) \int_{M}\left\|\beta_{V_{1}} \wedge \cdots \wedge \beta_{V_{p}}\right\|^{2} \leq \int_{M}\left\|\delta\left(\beta_{V_{1}} \wedge \cdots \wedge \beta_{V_{p}}\right)\right\|^{2} .
$$

Integrating with respect to $\left(\bar{V}_{1}, \ldots, \bar{V}_{p}\right) \in\left(\mathbf{S}^{d}\right)^{p}$ we then have, by Fubini's theorem:

$$
\begin{aligned}
& \mu_{1, p}^{\prime}(M) \int_{M} \int_{\left(\mathbf{S}^{d}\right)^{p}}\left\|\beta_{V_{1}} \wedge \cdots \wedge \beta_{V_{p}}\right\|^{2} d \bar{V}_{1} \ldots d \bar{V}_{p} d v_{M} \\
& \leq \int_{M} \int_{\left(\mathbf{S}^{d}\right)^{p}}\left\|\delta\left(\beta_{V_{1}} \wedge \cdots \wedge \beta_{V_{p}}\right)\right\|^{2} d \bar{V}_{1} \ldots d \bar{V}_{p} d v_{M},
\end{aligned}
$$

where we use the canonical measure on $\mathbf{S}^{d}$.

Note. To simplify the formulas below, we will replace the canonical measure $d \mu$ on $\mathbf{S}^{d}$ by its multiple $(d+1) / \operatorname{Vol}\left(\mathbf{S}^{d}\right) d \mu$. This change will not affect (2.3).

From now on we perform all calculations at a fixed point $x \in M$. If $\Phi$ is a self-adjoint endomorphism of $T_{x} M$, we extend it by derivations to a self-adjoint endomorphism $\Phi^{[p]}$ of $\Lambda_{x}^{p} M$. So:

$$
\Phi^{[p]} \omega\left(X_{1}, \ldots, X_{p}\right)=\sum_{j=1}^{p} \omega\left(X_{1}, \ldots, \Phi\left(X_{j}\right), \ldots, X_{p}\right)
$$

for all $p$-forms $\omega$ at $x$ and tangent vectors $X_{1}, \ldots, X_{p}$.

For $\nu \in T M^{\perp}$, let $S_{\nu} \in \operatorname{End}\left(T_{x} M\right)$ be the shape operator relative to $\nu$, defined by

$$
\left\langle S_{\nu}(X), Y\right\rangle=\langle L(X, Y), \nu\rangle,
$$

where $L(X, Y)$ is the second fundamental form of the immersion, and let $S_{\nu}^{[p]}$ be its (self-adjoint) extension acting on $\Lambda_{x}^{p} M$. For $p=1, \ldots, n$, denote by $T_{\nu}^{[p]}$ the endomorphism of $\Lambda_{x}^{p} M$ defined by

$$
T_{\nu}^{[p]}=S_{\nu}^{[p]}-n\langle H, \nu\rangle \cdot I
$$

and set $T_{\nu}^{[0]}=-n\langle H, \nu\rangle$. Note that $T_{\nu}^{[p]}$ is the extension to $\Lambda_{x}^{p} M$ of the endomorphism of $T_{x} M$ given by

$$
S_{\nu}-\frac{n}{p}\langle H, \nu\rangle \cdot I
$$


Finally, given an orthonormal basis $\left(\nu_{1}, \ldots, \nu_{m}\right)$ of $T_{x} M^{\perp}$, define

$$
\|S\|^{2}=\sum_{\alpha=1}^{m}\left\|S_{\nu_{\alpha}}\right\|^{2} \quad \text { and } \quad\left\|T^{[p]}\right\|^{2}=\sum_{\alpha=1}^{m}\left\|T_{\nu_{\alpha}}^{[p]}\right\|^{2} .
$$

For the proof of the next two lemmas we refer to the next section.

Lemma 2.1. At any point of $M$, one has

$$
\int_{\left(\mathbf{S}^{d}\right)^{p}}\left\|\beta_{V_{1}} \wedge \cdots \wedge \beta_{V_{p}}\right\|^{2} d \bar{V}_{1} \ldots d \bar{V}_{p}=p !\left(\begin{array}{c}
n \\
p
\end{array}\right) .
$$

Lemma 2.2. For all $p=1, \ldots, n$ one has, at any point of $M$ :

$$
\int_{\left(\mathbf{S}^{d}\right)^{p}}\left\|\delta\left(\beta_{V_{1}} \wedge \cdots \wedge \beta_{V_{p}}\right)\right\|^{2} d \bar{V}_{1} \ldots d \bar{V}_{p}=p !\left\|T^{[p-1]}\right\|^{2} .
$$

From Lemmas 2.1, 2.2 and formula (2.3), one then gets immediately:

Theorem 2.3. For all $p=1, \ldots, n$ :

$$
\mu_{1, p}^{\prime}(M) \leq \frac{1}{\operatorname{Vol}(M)} \int_{M}\left(\begin{array}{l}
n \\
p
\end{array}\right)^{-1}\left\|T^{[p-1]}\right\|^{2} .
$$

Remark 2.4. If $M$ is a closed hypersurface of $\mathbf{R}^{n+1}$, with principal curvatures $\eta_{1}, \ldots, \eta_{n}$, and if one defines the function $\Gamma_{p}$ on $M$ by

$$
\Gamma_{p}=\sum_{i_{1}<\cdots<i_{p}}\left(\eta_{i_{1}}+\cdots+\eta_{i_{p}}\right)^{2}
$$

then the upper bound of Theorem 2.3 is easily seen to take the form:

$$
\mu_{1, p}^{\prime}(M) \leq\left(\begin{array}{l}
n \\
p
\end{array}\right)^{-1} \frac{1}{\operatorname{Vol}(M)} \int_{M} \Gamma_{n-p+1} .
$$

Finally, we convert the right-hand side of the inequality in Theorem 2.3 into an expression involving only the square length of the mean curvature and the scalar curvature.

Lemma 2.5. For all $p=1, \ldots, n$ :

$$
\left\|T^{[p-1]}\right\|^{2}= \begin{cases}n^{2}\|H\|^{2} & \text { if } p=1, \\
\left(\begin{array}{l}
n-2 \\
p-2
\end{array}\right)\|S\|^{2}+\left(\begin{array}{c}
n-2 \\
p-1
\end{array}\right) n^{2}\|H\|^{2} & \text { if } p=2, \ldots, n-1, \\
\|S\|^{2} & \text { if } p=n .\end{cases}
$$

Proof. See the next section.

End of proof of Theorem [1.1] Using the Gauss lemma one verifies the identity:

$$
\|S\|^{2}=n^{2}\|H\|^{2}-\text { scal }
$$

and then, by Lemma 2.5 and a straightforward calculation:

$$
\left(\begin{array}{l}
n \\
p
\end{array}\right)^{-1} \cdot\left\|T^{[p-1]}\right\|^{2}=p n\|H\|^{2}-\frac{p(p-1)}{n(n-1)} \text { scal . }
$$


The inequality of Theorem 1.1 now follows from Theorem 2.3. By a calculation of Gallot-Meyer [G-M]:

$$
\mu_{1, p}^{\prime}\left(\mathbf{S}^{n}\right)=p(n-p+1)
$$

hence one has the equality for the canonical immersion of $\mathbf{S}^{n}$ into $\mathbf{R}^{n+1}$. Indeed, it is not difficult to verify that the forms defined in (2.2) are all eigenforms in that case (just differentiate formula (3.3)). The proof of Theorem 1.1 is now complete.

\section{Proof of Lemmas 2.1, 2.2, 2.5}

We use the following property of the fields $\bar{V}$ (also used in $[\underline{\mathrm{R}}$ ):

$$
\int_{\mathbf{S}^{d}}\langle\bar{V}, X\rangle\langle\bar{V}, Y\rangle d \bar{V}=\langle X, Y\rangle
$$

which is valid pointwise for all tangent vectors $X, Y \in T_{x} \mathbf{R}^{n+m}$, and where integration is taken with respect to $(d+1) / \operatorname{Vol}\left(\mathbf{S}^{d}\right)$-times the canonical measure on $\mathbf{S}^{d}$. The proof of (3.1) is straightforward using coordinates.

In what follows we fix a point $x \in M$, an orthonormal basis $\left(e_{1}, \ldots, e_{n}\right)$ of $T_{x} M$ and an orthonormal basis $\left(\nu_{1}, \ldots, \nu_{m}\right)$ of $T_{x} M^{\perp}$.

Proof of Lemma 2.1. We show more generally that, if $\Psi$ is an endomorphism of $\Lambda_{x}^{p} M$, then

$$
\int_{\left(\mathbf{S}^{d}\right)^{p}}\left\|\Psi\left(\beta_{V_{1}} \wedge \cdots \wedge \beta_{V_{p}}\right)\right\|^{2} d \bar{V}_{1} \ldots d \bar{V}_{p}=p !\|\Psi\|^{2} .
$$

Lemma 2.1 will follow by applying (3.2) to the identity operator of $\Lambda_{x}^{p} M$, whose squared norm is in fact $\left(\begin{array}{l}n \\ p\end{array}\right)$.

To prove (3.2), let $\left(\xi_{1}, \ldots, \xi_{n}\right)$ be the dual basis of $\left(e_{1}, \ldots, e_{n}\right)$. Then

$$
\beta_{V_{1}}=\sum_{j=1}^{n}\left\langle V_{1}, e_{j}\right\rangle \xi_{j}=\sum_{j=1}^{n}\left\langle\bar{V}_{1}, e_{j}\right\rangle \xi_{j},
$$

and one has

$$
\begin{aligned}
\int_{\left(\mathbf{S}^{d}\right)^{p}} & \left\|\Psi\left(\beta_{V_{1}} \wedge \cdots \wedge \beta_{V_{p}}\right)\right\|^{2} \bar{V}_{1} \ldots d \bar{V}_{p} \\
= & \int_{\left(\mathbf{S}^{d}\right)^{p}}\left\|\sum_{j}\left\langle\bar{V}_{1}, e_{j}\right\rangle \Psi\left(\xi_{j} \wedge \beta_{V_{2}} \wedge \cdots \wedge \beta_{V_{p}}\right)\right\|^{2} d \bar{V}_{1} \ldots d \bar{V}_{p} \\
= & \sum_{j} \int_{\left(\mathbf{S}^{d}\right)^{p-1}}\left\|\Psi\left(\xi_{j} \wedge \beta_{V_{2}} \wedge \cdots \wedge \beta_{V_{p}}\right)\right\|^{2} d \bar{V}_{2} \ldots d \bar{V}_{p} .
\end{aligned}
$$

Note that the second inequality follows because, by (3.1):

$$
\int_{\mathbf{S}^{d}}\left\langle\bar{V}_{1}, e_{j}\right\rangle\left\langle\bar{V}_{1}, e_{k}\right\rangle d \bar{V}_{1}=\delta_{j k}
$$

By induction,

$$
\int_{\left(\mathbf{S}^{d}\right)^{p}}\left\|\Psi\left(\beta_{V_{1}} \wedge \cdots \wedge \beta_{V_{p}}\right)\right\|^{2} d \bar{V}_{1} \ldots d \bar{V}_{p}=\sum_{j_{1}, \ldots, j_{p}}\left\|\Psi\left(\xi_{j_{1}} \wedge \cdots \wedge \xi_{j_{p}}\right)\right\|^{2}=p !\|\Psi\|^{2} .
$$


Proof of Lemma [2.2] A straightforward argument shows that $\nabla \beta_{V}$ is the second fundamental form in the direction $V^{\perp}$ :

$$
\nabla_{e_{j}} \beta_{V}\left(e_{k}\right)=\left\langle S_{V^{\perp}}\left(e_{j}\right), e_{k}\right\rangle .
$$

In particular,

$$
\delta \beta_{V}=-n\left\langle H, V^{\perp}\right\rangle .
$$

We will prove below that, for $p \geq 2$, the following formula holds:

$$
\begin{aligned}
\delta\left(\beta_{V_{1}} \wedge \cdots \wedge \beta_{V_{p}}\right) & =\sum_{i=1}^{p}(-1)^{i+1} T_{V_{i}^{\perp}}^{[p-1]}\left(\beta_{V_{1}} \wedge \cdots \wedge \hat{\beta}_{V_{i}} \wedge \cdots \wedge \beta_{V_{p}}\right) \\
& =\sum_{i=1}^{p}(-1)^{i+1} T_{V_{i}^{\perp}}^{[p-1]}\left(\hat{\beta}_{i}\right),
\end{aligned}
$$

where we have set

$$
\hat{\beta}_{i}=\beta_{V_{1}} \wedge \cdots \wedge \hat{\beta}_{V_{i}} \wedge \cdots \wedge \beta_{V_{p}}
$$

and where $T_{\nu}^{[p]}=S_{\nu}^{[p]}-n\langle H, \nu\rangle \cdot I$ as in 2.4 .

Given (3.3) one proves Lemma 2.2 as follows:

$$
\begin{aligned}
\int_{\left(\mathbf{S}^{d}\right)^{p}} & \left\|\delta\left(\beta_{V_{1}} \wedge \cdots \wedge \beta_{V_{p}}\right)\right\|^{2} d \bar{V}_{1} \ldots d \bar{V}_{p} \\
= & \sum_{i=1}^{p} \int_{\left(\mathbf{S}^{d}\right)^{p}}\left\|T_{V_{i}^{\perp}}^{[p-1]}\left(\hat{\beta}_{i}\right)\right\|^{2} d \bar{V}_{1} \ldots d \bar{V}_{p} \\
= & p \int_{\left(\mathbf{S}^{d}\right)^{p}}\left\|T_{V_{1}^{\perp}}^{[p-1]}\left(\beta_{V_{2}} \wedge \cdots \wedge \beta_{V_{p}}\right)\right\|^{2} d \bar{V}_{1} d \bar{V}_{2} \ldots d \bar{V}_{p} \\
= & p \sum_{\alpha=1}^{m} \int_{\left(\mathbf{S}^{d}\right)^{p-1}}\left\|T_{\nu_{\alpha}}^{[p-1]}\left(\beta_{V_{2}} \wedge \cdots \wedge \beta_{V_{p}}\right)\right\|^{2} d \bar{V}_{2} \ldots d \bar{V}_{p} \\
= & p(p-1) ! \sum_{\alpha=1}^{m}\left\|T_{\nu_{\alpha}}^{[p-1]}\right\|^{2} \\
= & p !\left\|T^{[p-1]}\right\|^{2} .
\end{aligned}
$$

In fact, the first equality follows because, for fixed forms $\omega, \phi$ at $x$ and $\nu \in T_{x} M^{\perp}$ one has, by (3.1) and linearity:

$$
\int_{\mathbf{S}^{d}}\left\langle T_{V^{\perp}}^{[p-1]}(\omega), T_{\nu}^{[p-1]}\left(\beta_{V} \wedge \phi\right)\right\rangle d \bar{V}=0,
$$

which implies

$$
\int_{\left(\mathbf{S}^{d}\right)^{p}}\left\langle T_{V_{i}^{\perp}}^{[p-1]}\left(\hat{\beta}_{i}\right), T_{V_{j}^{\perp}}^{[p-1]}\left(\hat{\beta}_{j}\right)\right\rangle d \bar{V}_{1} \ldots d \bar{V}_{p}=0 \quad \text { if } \quad i \neq j ;
$$

the second equality is true because all terms in the sum are equal; the third holds because, for all $(p-1)$-forms $\omega$ :

$$
\int_{\mathbf{S}^{d}}\left\|T_{V^{\perp}}^{[p-1]}(\omega)\right\|^{2} d \bar{V}=\sum_{\alpha=1}^{m}\left\|T_{\nu_{\alpha}}^{[p-1]}(\omega)\right\|^{2} ;
$$

the fourth equality follows from (3.2) applied to $\Psi=T_{\nu_{\alpha}}^{[p-1]}$, and the fifth is definition (2.6). 
It remains to prove (3.3). Pick any $p-1$ vectors, say $e_{1}, \ldots, e_{p-1}$ for simplicity, from the orthonormal basis $\left(e_{1}, \ldots, e_{n}\right)$ of $T_{x} M$. Then:

$$
\begin{aligned}
& -\delta\left(\beta_{V_{1}} \wedge \cdots \wedge \beta_{V_{p}}\right)\left(e_{1}, \ldots, e_{p-1}\right) \\
= & \sum_{k=1}^{n} \nabla_{e_{k}}\left(\beta_{V_{1}} \wedge \cdots \wedge \beta_{V_{p}}\right)\left(e_{k}, e_{1}, \ldots, e_{p-1}\right) \\
= & \sum_{k=1}^{n} \sum_{i=1}^{p-1}(-1)^{i+1}\left[\nabla_{e_{k}} \beta_{V_{i}}\right] \wedge \beta_{V_{1}} \wedge \cdots \wedge \hat{\beta}_{V_{i}} \wedge \cdots \wedge \beta_{V_{p}}\left(e_{k}, e_{1}, \ldots, e_{p-1}\right) \\
(3.4)= & \sum_{k=1}^{n} \sum_{i=1}^{p-1}(-1)^{i+1}\left[\nabla_{e_{k}} \beta_{V_{i}}\right] \wedge \hat{\beta}_{i}\left(e_{k}, e_{1}, \ldots, e_{p-1}\right) .
\end{aligned}
$$

Fix $i=1, \ldots, p-1$. Then:

$$
\begin{aligned}
& {\left[\nabla_{e_{k}} \beta_{V_{i}}\right] \wedge \hat{\beta}_{i}\left(e_{k}, e_{1}, \ldots, e_{p-1}\right) } \\
= & \nabla_{e_{k}} \beta_{V_{i}}\left(e_{k}\right) \hat{\beta}_{i}\left(e_{1}, \ldots, e_{p-1}\right)+\sum_{j=1}^{p-1}(-1)^{j} \nabla_{e_{k}} \beta_{V_{i}}\left(e_{j}\right) \hat{\beta}_{i}\left(e_{k}, e_{1}, \ldots, \hat{e}_{j}, \ldots, e_{p-1}\right) \\
= & \left\langle S_{V_{i}^{\perp}}\left(e_{k}\right), e_{k}\right\rangle \hat{\beta}_{i}\left(e_{1}, \ldots, e_{p-1}\right) \\
& +\sum_{j=1}^{p-1}(-1)^{j}\left\langle S_{V_{i}^{\perp}}\left(e_{k}\right), e_{j}\right\rangle \hat{\beta}_{i}\left(e_{k}, e_{1}, \ldots, \hat{e}_{j}, \ldots, e_{p-1}\right) .
\end{aligned}
$$

Summing over $k$ :

$$
\begin{aligned}
& \sum_{k=1}^{n}\left[\nabla_{e_{k}} \beta_{V_{i}}\right] \wedge \hat{\beta}_{i}\left(e_{k}, e_{1}, \ldots, e_{p-1}\right) \\
= & n\left\langle H, V_{i}^{\perp}\right\rangle \hat{\beta}_{i}\left(e_{1}, \ldots, e_{p-1}\right)+\sum_{j=1}^{p-1}(-1)^{j} \hat{\beta}_{i}\left(S_{V_{i}^{\perp}}\left(e_{j}\right), e_{1}, \ldots, \hat{e}_{j}, \ldots, e_{p-1}\right) \\
= & n\left\langle H, V_{i}^{\perp}\right\rangle \hat{\beta}_{i}\left(e_{1}, \ldots, e_{p-1}\right)-\sum_{j=1}^{p-1} \hat{\beta}_{i}\left(e_{1}, \ldots, S_{V_{i}^{\perp}}\left(e_{j}\right), \ldots, e_{p-1}\right) \\
= & n\left\langle H, V_{i}^{\perp}\right\rangle \hat{\beta}_{i}\left(e_{1}, \ldots, e_{p-1}\right)-S_{V_{i}^{\perp}}^{[p-1]}\left(\hat{\beta}_{i}\right)\left(e_{1}, \ldots, e_{p-1}\right) \\
= & -T_{V_{i}^{\perp}}^{[p-1]}\left(\hat{\beta}_{i}\right)\left(e_{1}, \ldots, e_{p-1}\right) .
\end{aligned}
$$

Inserting in (3.4) we have

$$
-\delta\left(\beta_{V_{1}} \wedge \cdots \wedge \beta_{V_{p}}\right)\left(e_{1}, \ldots, e_{p-1}\right)=-\sum_{i=1}^{p-1}(-1)^{i+1} T_{V_{i}^{\perp}}^{[p-1]}\left(\hat{\beta}_{i}\right)\left(e_{1}, \ldots, e_{p-1}\right)
$$

which proves 3.3 .

Proof of Lemma 2.5. Let $F$ be a Euclidean vector space of dimension $n$, and $\Phi$ a self-adjoint endomorphism of $F$. Then, if $\Phi^{[p]}$ is the extension of $\Phi$ acting on $\Lambda^{p}(F)$, one has

$$
\left\|\Phi^{[p]}\right\|^{2}=\left(\begin{array}{c}
n-2 \\
p-1
\end{array}\right)\|\Phi\|^{2}+\left(\begin{array}{c}
n-2 \\
p-2
\end{array}\right)(\operatorname{tr} \Phi)^{2},
$$


by a straightforward verification (by convention, $\left(\begin{array}{l}a \\ b\end{array}\right)=0$ whenever $b>a$ or $b<0$ ).

We take $F=T_{x} M, \nu \in T_{x} M^{\perp}$ and $\Phi=S_{\nu}-\frac{n}{p}\langle H, \nu\rangle I$, so that $\Phi^{[p]}=T_{\nu}^{[p]}$ (see (2.5)). Since $\operatorname{tr} S_{\nu}=n\langle H, \nu\rangle$ :

$$
\left\|T_{\nu}^{[p]}\right\|^{2}=\left(\begin{array}{c}
n-2 \\
p-1
\end{array}\right)\left\|S_{\nu}\right\|^{2}+\left(\begin{array}{c}
n-2 \\
p
\end{array}\right) n^{2}\langle H, \nu\rangle^{2} .
$$

Changing $p$ to $p-1$, and summing over the vectors of an orthonormal basis of $T_{x} M^{\perp}$, we get the final assertion.

\section{REFERENCES}

[B-W] Bleecker D., Weiner, J. Extrinsic bounds on $\lambda_{1}$ of $\Delta$ on a compact manifold, Comment. Math. Helv. 51 (1976) 601-609 MR 0425839 (54:13789)

[C] Chavel, I. Eigenvalues in Riemannian Geometry (Appendix by J. Dodziuk), Academic Press, Inc. 1984 MR 0768584 (86g:58140)

[G-M] Gallot, S., Meyer, D. Opérateur de courbure et Laplacien des formes différentielles d'une variété riemannienne, J. Math. Pures Appl. 54 (1975) 259-284 MR 0454884 (56:13128)

[G-S] Guerini, P., Savo, A. Eigenvalue and gap estimates for the Laplacian acting on p-forms, Trans. Amer. Math. Soc. 356 (2004), 319-344 MR 2020035

[R] Reilly, R.C. On the first eigenvalue of the Laplacian for compact submanifolds of Euclidean space, Comment. Math. Helv. 52, n. 4 (1977) 525-533 MR 0482597 (58:2657)

Dipartimento di Metodi e Modelli Matematici, Università di Roma, la Sapienza, Via Antonio Scarpa 16, 00161 Roma, Italy

E-mail address: savo@dmmm.uniroma1.it 